\title{
A Single Image Registration Method for CT Guided Interventions
}

\author{
Robert C. Susil ${ }^{1}$, James H. Anderson ${ }^{2}$, and Russell H. Taylor ${ }^{3}$ \\ ${ }^{1}$ Department of Biomedical Engineering \\ ${ }^{2}$ Department of Radiology \\ ${ }^{3}$ Department of Computer Science \\ The Johns Hopkins University, 3400 N. Charles Street, Baltimore, Maryland 21218 \\ rht@cs.jhu.edu
}

\begin{abstract}
Minimally invasive image guided interventions are an attractive option for localized therapy delivery and diagnostic biopsy. We have developed a method for CT guided needle placement, based upon the BrownRoberts-Wells frame, which requires no immobilization or fiducial implantation. A localization module, placed on a needle holding robotic end effector, allows for localization of the effector in the image space using a single CT image. In a theoretical analysis, we show that this registration method has attractive sensitivity and error attenuation properties. Experimentally, the average error in needle tip location over 63 trials was $470 \mu \mathrm{m} ; 95 \%$ of the errors were below $1.0 \mathrm{~mm}$. This method is a fast, accurate, and easily implemented registration method for cross sectional image guided stereotaxis.
\end{abstract}

\section{Objective}

\subsection{Motivation}

Recent advances have identified a variety of novel anticancer therapeutic agents and targets. However, significant obstacles still hinder the effective delivery of these therapies to target tumor sites [1]. As cancer is expected to surpass cardiovascular disease as the major cause of death in the United States within five years [2], effective solutions to these delivery problems are warranted.

One solution to this delivery problem is to physically place therapeutic agents in or near the tumor site. However, for neural or visceral tumors, the physical delivery of therapy to the tumor is inherently a stereotactic problem. Therefore, effective image guided methods are needed to facilitate the accurate placement of therapy. In addition, these same methods can be applied to tumor biopsy for diagnostic tests.

Computed Tomography is a popular diagnostic imaging modality that is often used for the visualization of tumors. While CT provides high resolution cross sections of the anatomy, few techniques exist which can easily integrate this information with 
percutaneous therapy delivery to soft tissues. Extensive past efforts have been made to use CT images for guidance during biopsy and therapy for intracranial lesions [3] However, these methods largely involve the fixation of a stereotactic frame to the patient's skull, a significantly invasive procedure. For procedures involving soft tissue, such as within in the abdominal cavity, attaching a stereotactic frame is not feasible. Therefore, it would be preferable to have a method for CT guided tissue biopsy and therapy delivery which could obviate the need to physically attach a stereotactic frame to the patient.

\subsection{Solution Method}

We have applied a localization method that allows for the guidance of stereotactic procedures using single CT image slices. Instead of attaching a frame to the patient, a localization module, based on the Brown-Roberts-Wells frame $[4,5]$, is attached to a needle holding robotic end effector (Figure 1). Due to the localization module's fiducial pattern, a single cross sectional image allows us to determine the pose of the needle in the image space. Instead of determining the position of the lesion in some external reference frame, we simply find the biopsy needle in the image coordinate system, along with the anatomy. Therefore, in a single image slice that intersects the target lesion and our needle localization module, we have enough information to determine the necessary kinematics to guide the needle to the target.

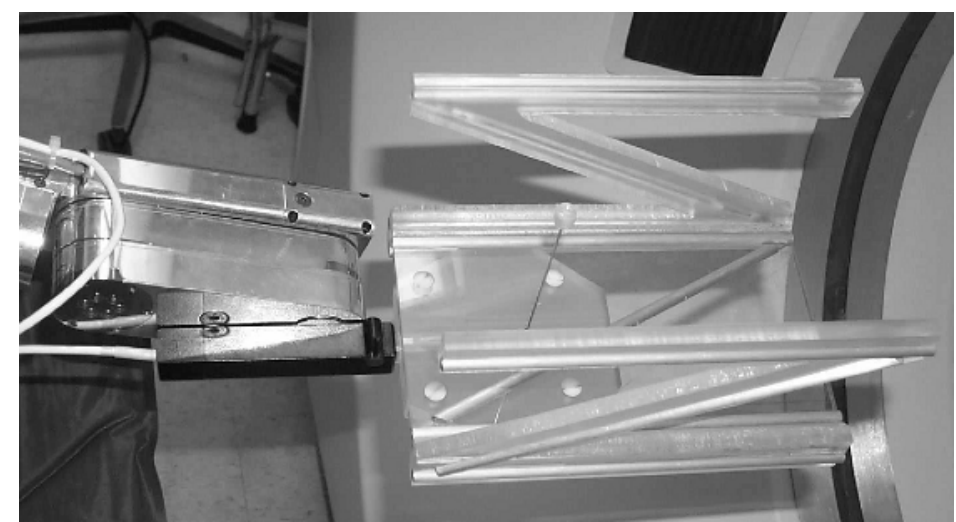

Figure 1: Localization module attached to the end effector. When inserted into the image field of view, a cross section of each of the nine aluminum fiducial bars appears in the CT image, allowing for registration.

\subsection{Current Aims}

Our final goal is complete automation of the needle placement, using a robotic arm, once the target is identified by the physician. However, the currently reported work focuses on the development and testing of the needle localization module itself. First, we show that the localization motif is theoretically robust over a range of poses, having both sensitivity to small positional changes and relative insensitivity to 
measurement errors. Subsequently, we compare our ability to determine the needle pose using single CT image slices with an independent multiple slice pose determination method, which we show to be an accurate ground truth. For $63 \mathrm{CT}$ images, the average net displacement error at the needle tip (located $10 \mathrm{~cm}$ from the center of the needle holder) was $630 \mu \mathrm{m}$ and $95 \%$ of the errors were under $1.0 \mathrm{~mm}$. This localization scheme, which still can be improved upon with minor modifications, is therefore shown to be both simple and accurate.

\subsection{Prior Work and Present Contribution}

Percutaneous procedures require one to determine the position of an internal target without direct visualization. Most often, this involves registration of an image data set, in which the target is identified, with physical space. This procedure, stereotaxy, was founded by Clarke and Horsley in 1906 [6]. Most techniques have been based upon an rigid frame which is attached to the patient, providing a common coordinate system through which the image and physical spaces can be related [3]. While stereotactic procedures were initially advanced using two-dimensional imaging modalities, the advent of Computed Tomography in the 1970's greatly accelerated development and application. Instead of projecting three-dimensional structures into two-dimensions, this modality provides a series of 2D image slices, allowing for true three-dimensional reconstruction.

The Brown-Roberts-Wells (BRW) frame was first introduced in 1979 [4]. The frame, consisting of three $\mathrm{N}$ shaped motifs attached to the patient's skull, represented a major advance in CT localization. Previous frames were constrained to remain strictly perpendicular to the image plane, providing little flexibility [3]. However, the BRW frame was more versatile in that the position and orientation of the frame was fully encoded within each image slice, allowing for rotations and tilting of the frame relative to the image plane[4]. Therefore, the position of any point can easily be found in both the frame and the image space coordinate systems.

We have applied this fiducial motif with several additions. Primarily, instead of fixing the frame to the patient, we have placed the frame on our end effector. Therefore, while we are still able to find the relation between the patient's anatomy and the end effector with a singe CT slice, the procedure is markedly less invasive. Moreover, in previous applications, the base ring of the $\mathrm{N}$-frame fiducials remained nearly parallel to the image plane.[7-9]. However, because the frame is now placed on the mobile end effector (Figure 1), we need to ensure that this localization scheme is robust over a range of operating points. Therefore, we perform a theoretical analysis, examining both sensitivity to small positional changes and attenuation of measurement errors. In addition, we experimentally compare this single image registration method with an independent multislice registration method.

Many techniques have been developed which integrate robotic guidance of end effectors with image based stereotactic procedures using a variety of registration techniques. For example, Lavallee et al. implemented a system for image guided intracranial needle placement using biplaner x-ray [10]. In another neurosurgical system, Kwoh et al. develop a surgical plan using multiple CT image slices, register by docking their robot with the patient's stereotactic head frame, and then place the needle without CT surveillance[11]. Glauser et al. also use a stereotactic head frame 
to register the robot and image space, but are able to perform needle placement under active CT surveillance to confirm the position of their end effector [12]. Similarly, Masamune et al. perform needle placement within the CT scanner and register using a stereotactic head frame [13]. More recently, Bzostek et al. have developed a technique for stereotactic procedures, under biplanar fluoroscopy, in order to access mobile organs (e.g. the kidneys) $[14,15]$.

Similarly, our technique is designed to facilitate end effector placement within the CT scanner. However, while previous techniques have relied on techniques that only register the robot space once, we are able to perform our registration with every image slice. Primarily, the technique is simple, requiring no extensive calibration routine. Moreover, it allows for immediate confirmation of the end effector position with each slice and therefore, the system is robust and well suited for dynamic and error prone situations.

\subsection{Target Application Example}

Although the applications of this localization scheme are numerous, an obvious application is for percutaneous tissue biopsy within the abdominal cavity. First, the patient is placed in the CT scanner and a complete set of images in the area of interest is collected. Next, upon recognition of a tumor mass, the physician selects a biopsy target and a skin entry site from the image set. The robot, with biopsy needle end effector and localization module, is positioned such that the localization module is within the imager field of view. Next, a single image is taken, containing both the biopsy target and a cross section of the localization module. From this one image, the necessary translation and rotation to reach the target is determined and subsequently executed by the robot. The robot, or an attendant surgeon, can then drive the biopsy needle. Because the patient remains within the scanner, a single image will confirm that the needle has reached the target site. The biopsy is then taken, completing the procedure quickly and with minimal invasiveness. We emphasize that this system, with minor material modifications, can easily be extended to use with MRI.

\section{Methods}

\subsection{Description of Registration Method}

In order to register the biopsy needle to the image space, we need to find a set of three corresponding points in both the needle holder coordinate system, $H$, and the image coordinate system, $I$. These three points define a coordinate system $P$. By finding the position and orientation of $P$ in the image, ${ }_{P}^{I} T$, and the holder space, ${ }_{P}^{H} T$, we can then determine the pose of the holder in the image coordinate system, ${ }_{H}^{I} T={ }_{P}^{I} T\left({ }_{P}^{H} T\right)^{-1}$. Having previously performed the calibration of the needle holder, $H$, and the needle, $N$, coordinate systems, ${ }_{N}^{H} T$, we can find ${ }_{N}^{I} T$, the pose of the biopsy needle in the image space. 
In order to find the set of three corresponding points in both the image and holder coordinate systems using only one CT image, we use the Brown-Roberts-Wells frame.

\subsection{Description of Holder}

The localization module of the needle holder is illustrated in Figure 1. The module is designed to easily attach to a needle driver end effector $[16,17]$ using four screws. The basic structural element of the module is the ' $N$ ' shaped fiducial motif (Figure 2a). This motif is repeated three times, forming a ' $U$ ' shaped module with one fiducial
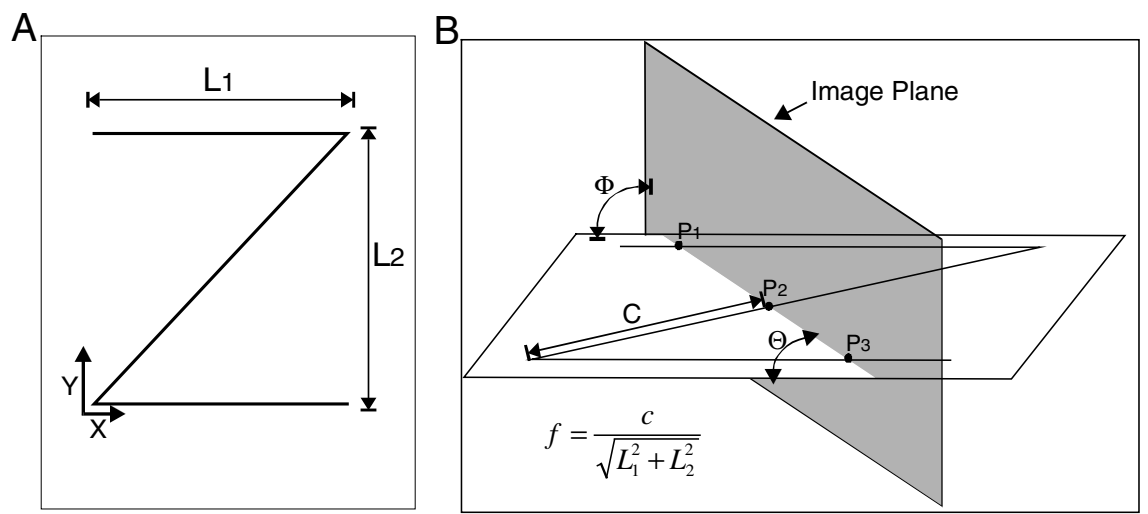

Figure 2: Panel A: Dimensioning and coordinate system conventions for the fiducial motifs. Panel B: One fiducial motif intersected by the image plane. $p_{1}, p_{2}$, and $p_{3}$ are the three fiducial bar points of intersection with the image plane. $f, \phi$, and $\theta$ define

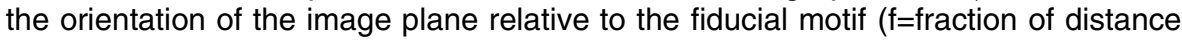
along diagonal fiducial where intersection occurs).

motif as a base and the other two fiducial motifs as sides (Figure 1).

The fiducial lines in each motif are made of 0.25 inch diameter aluminum rod inlaid in acrylic. In our prototype, $\mathrm{L}_{1}=8$ inches and $\mathrm{L}_{2}=4$ inches (Figure $2 \mathrm{a}$ ). We define a holder coordinate system, $H$, with an origin at the center of the ' $U$ ' shaped module and an orientation coincident with that of the base plate fiducial motif (Figure 2a).

\subsection{Single Image Registration Method}

A CT image of each fiducial motif produces a cross section of the three bars, yielding three ellipses in the image. By finding the centroids of these ellipses we can locate the centers of the three bars where they intersect the image plane. Using these three points, ${ }^{\mathrm{I}} p_{1},{ }^{\mathrm{I}} p_{2}$, and ${ }^{\mathrm{I}} p_{3}$, we can determine the position of one corresponding point, $c p_{\mathrm{n}}$, in both the holder space, ${ }^{\mathrm{H}} c p_{\mathrm{n}}$, and the image space, ${ }^{\mathrm{I}} c p_{\mathrm{n}}$.

We can describe the relationship between the fiducial motif and the image plane with three parameters: $f$, the fraction of the distance along the diagonal fiducial where 
the intersection occurs; $\phi$, the angle between the fiducial motif plane and the image plane; and $\theta$, the angle between the parallel fiducial bars and the line of intersection (Figure 2b).

The distances $\left|{ }^{\mathrm{FM}} p_{1^{-}}{ }^{\mathrm{FM}} p_{2}\right|$ and $\left|{ }^{\mathrm{FM}} p_{3^{-}}{ }^{\mathrm{FM}} p_{2}\right|$, expressed as a function of $f, \phi$, and $\theta$, are:

$$
\begin{aligned}
& \left|{ }^{F M} p_{1}-{ }^{F M} p_{2}\right|=\csc (\theta) L_{2}(1-f) \\
& \left|{ }^{F M} p_{3}-{ }^{F M} p_{2}\right|=\csc (\theta) L_{2}(f)
\end{aligned}
$$

The ratio of these distances is:

$$
\frac{\left|{ }^{F M} p_{1}-{ }^{F M} p_{2}\right|}{\left|{ }^{F M} p_{3}-{ }^{F M} p_{2}\right|}=\frac{1}{f}-1
$$

This ratio is only a function of $f$, the fraction of the distance along the diagonal fiducial where the intersection occurs. Because the transformation from the fiducial motif coordinate system to the image space is a rigid body transformation, we can determine the point where the image plane intersects the diagonal bar, ${ }^{\mathrm{FM}} p_{2}$, by finding the ratio of the distances between points ${ }^{\mathrm{I}} p_{1},{ }^{\mathrm{I}} p_{2}$, and ${ }^{\mathrm{I}} p_{3}$. From a previous calibration of our holder, we know the transformation for this point, ${ }^{\mathrm{FM}} p_{2}$, to the holder coordinate system, ${ }_{F M}^{H} T$. Therefore, we know the position of this intersection in both the image space, ${ }^{\mathrm{I}} p_{2}$, and the holder space, ${ }^{\mathrm{H}} p_{2}$, providing one of the three corresponding points, $c p_{1}$. We repeat this method for the two remaining fiducial motifs, generating all three corresponding points, $c p_{1}, c p_{2}$, and $c p_{3}$.

\subsection{Error Analysis}

With the set of three points generated by the intersection of each of the three fiducial motifs and the image plane, we could do more than determine one corresponding point in the image and holder coordinate systems. Namely, we could also determine the angle $\theta$ (Figure 2b). However, we have very little accuracy in determining this angle. When operating about $\theta=90^{\circ}$, the sensitivity of our assessment of $\theta\left(\partial \theta_{\text {measured }} / \partial \theta_{\text {actual }}\right)$ is zero. In contrast, determination of the corresponding point has much more attractive error properties, which we demonstrate here.

To robustly determine the corresponding points, the localization method must have two properties. First, the assessment of ${ }^{\mathrm{H}} c p_{\mathrm{n}}$ should be relatively insensitive to small measurement errors in $\left|p_{1}^{\mathrm{I}}-{ }^{\mathrm{I}} p_{2}\right|$ and $\left|{ }^{\mathrm{I}} p_{3}-{ }^{\mathrm{I}} p_{2}\right|$. These sensitivities to measurement error are:

$$
\frac{\partial c}{\partial\left|{ }^{I} p_{1}-{ }^{I} p_{2}\right|_{\text {measured }}}=-f \sqrt{1+\left(\frac{L_{1}}{L_{2}}\right)^{2}} \sin (\theta)
$$




$$
\frac{\partial c}{\partial\left|{ }^{I} p_{3}-{ }^{I} p_{2}\right|_{\text {measured }}}=(1-f) \sqrt{1+\left(\frac{L_{1}}{L_{2}}\right)^{2}} \sin (\theta)
$$

Near the operating point $\left(\theta=90^{\circ}\right.$ and $\left.f=0.5\right)$, the magnitudes of the sensitivities are 0.71 . As $\theta$ decreases, the system becomes less sensitive to measurement errors. The worst case measurement error sensitivity is 1.41. However, we can improve these values by decreasing the $L_{1} / L_{2}$ ratio (see Future Work).

Second, the measured parameters, $\left|p_{1}^{\mathrm{I}} p_{-}{ }^{\mathrm{I}} p_{2}\right|$ and $\left|{ }^{\mathrm{I}} p_{3}-{ }^{\mathrm{I}} p_{2}\right|$, must be sensitive to small changes in $c$, the distance from the image plane intersection with the diagonal fiducial to the fiducial motif origin (i.e. $c=f \sqrt{L_{1}^{2}+L_{2}^{2}}$ ) (Figure 2b). This sensitivity is:

$$
\begin{aligned}
& \frac{\partial\left|{ }^{I} p_{1}-{ }^{I} p_{2}\right|}{\partial c}=-\csc (\theta) / \sqrt{1+\left(\frac{L_{1}}{L_{2}}\right)^{2}} \\
& \frac{\partial\left|{ }^{I} p_{3}-{ }^{I} p_{2}\right|}{\partial c}=\csc (\theta) / \sqrt{1+\left(\frac{L_{1}}{L_{2}}\right)^{2}}
\end{aligned}
$$

At the operating point of $\theta=90^{\circ}$, the magnitudes of the sensitivities are 0.71 , which is the worst case for the system. As $\theta$ decreases, the sensitivity increases. Also, as was the case previously, we can improve the sensitivity by decreasing the $L_{1} / L_{2}$ ratio (see Future Work).

In summary, we find that the system has relatively good error characteristics. The worst case sensitivity to measurement error is 1.41 and the worst case sensitivity of the system is 0.71 (for $L_{1} / L_{2}=1$ ).

\subsection{Multislice Registration Method}

In order to determine the accuracy of the single slice registration method, we need to determine the ground truth pose of the holder in the image space. To do this, we performed a multislice registration of the holder. By using several image slices, we can find a series of points along each fiducial bar in the image coordinate system. By performing a least squares line fit, we can very accurately determine the pose of the holder in the image space.

The accuracy of this registration method was assessed by comparing the calculated transformations between the three fiducial motif coordinate systems which compose the localization module. As these transformations are determined by the geometry of the module (Figure 1), they are invariant. Over the six image sets studied, the calculated transformations had an average variation of $0.066^{\circ}$ and $120 \mu \mathrm{m}$. Therefore, we are confident in using this multislice registration method as our ground truth. 


\section{Results}

\subsection{Experiment Design}

To determine the accuracy of our single slice localization method, we compared the single slice determination of the needle pose, ${ }_{N}^{I} T_{S S}$, with the multislice ground truth determination of needle pose, ${ }_{N}^{I} T_{M S}$. An average of 13 images were obtained with the holder in each of 5 different poses (a total of 63 images). All images were obtained in a GE Genesis CT Scanner. Image slices were $5 \mathrm{~mm}$ thick and the image pixels were $0.7 \mathrm{~mm}$ by $0.7 \mathrm{~mm}$.

\subsection{Results}

Error is defined as the difference between the multislice determined ground truth and the single slice determined pose. Components include angular error of holder pose and offset error of holder pose. From these two components, net displacement error at the needle tip, $10 \mathrm{~cm}$ from the center of the holder, was found. The average angular error was $0.32^{\circ}$, the average displacement offset error was $380 \mu \mathrm{m}$, and the average displacement error at the needle tip was $630 \mu \mathrm{m}$. Figure 3 presents the displacement error probability density function with a best-fit gamma distribution ( $\lambda=2.95$ and $\alpha=0.16$ ). $95 \%$ of the needle tip displacement errors were below $1.0 \mathrm{~mm}$ and the maximum error seen in the 63 images was $1.45 \mathrm{~mm}$.

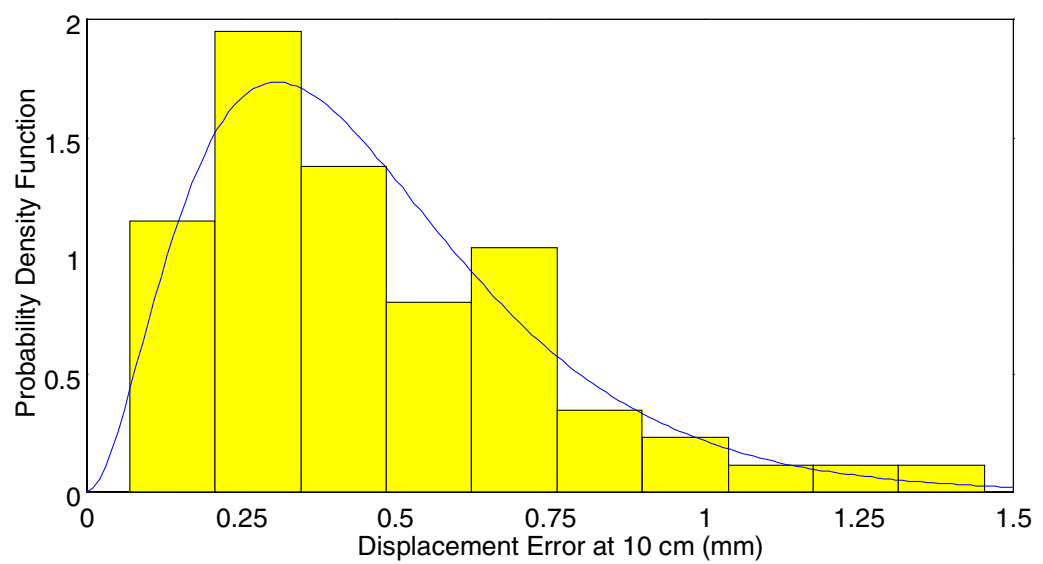

Figure 3: Experimental data and the best fit, by maximum likelihood, gamma distribution $(\lambda=2.95$ and $\alpha=0.16)$. $95 \%$ of the errors are found below $1.0 \mathrm{~mm}$. 


\section{Discussion}

We have developed a system, based upon the Brown-Roberts-Wells frame, that allows for accurate determination of end effector pose using a single CT image. While this study was carried out entirely under CT, the same methods can be applied to other cross sectional imaging modalities, namely, MRI.

Most notably, we find that the localization frame provides accurate registration over a range of operating points. In previous work, it was found that the BrownRoberts-Wells was accurate enough to be applied in neurosurgical interventions [6-8] However, these applications employed a narrow operating range with the stereotactic base ring nearly parallel to the scan plane. Here, because the frame is attached to the robotic end-effector, we have a very large operating range. Even over this large range, both our theoretical and experimental error analysis show that the system is accurate and reliable.

We emphasize several positive features of this system. First, it is minimally invasive. There is no need to attach any fiducial frames or markers to the patient, making it ideal for soft tissue interventions where attachment of a stereotactic frame is not practical. Second, it allows for real time confirmation of needle position relative to the anatomy. Because the whole system is integrated within the CT scanner, images can be taken at any point, such as for positive confirmation of needle placement within a lesion. Finally, the system is relatively simple in that it requires no external reference frames. All registration is done based upon single CT images. While work remains to be done in order to integrate this system with a robotic actuator and on-line target selection, our localization module is a significant first step in developing a versatile, integrated image guided stereotactic system.

\section{Future Work}

Although we achieved a good level of accuracy in the present study, there are several ways in which our system can be more robust.

\subsection{Multiple Resolution Fiducial Motifs}

As discussed in the Methods section, the localization system's sensitivity is dependent upon minimizing the ratio $L_{1} / L_{2}$ (Figure 2 and Equations 4-7). That is, the steeper the diagonal fiducial bar, the better. In the present study, $L_{1} / L_{2}=2$. While this may not seem optimal, a high $L_{1} / L_{2}$ ratio gives us a larger range of valid image slices. In order for the localization module to work, all three fiducial motifs, therefore all nine fiducial bars, must intersect the image plane. While a low $L_{1} / L_{2}$ ratio yields better sensitivity, it also shortens the localization module, resulting in only a few valid image slices. Therefore, we must reach a balance between a low ratio for good sensitivity and a higher ratio for a larger number of valid images.

In order to obtain both of these benefits, we could place multiple fiducial motifs on each of the three planes. This would appear as several diagonal fiducial bars, one with a small slope and several with large slopes, on each plane. The high $L_{1} / L_{2}$ ratio motif (i.e. small slope) could provide an initial pose estimate while a smaller $L_{1} / L_{2}$ ratio motif (i.e. larger slope) could then provide more accurate pose determination. 


\subsection{Centroid Finding Method}

In the present work, we used a simple centroid finding routine in order to find the centers of our fiducial bars in the CT image. The images were thresholded and binarized, removing all data except for the locations of the aluminum fiducial bars. To find the centroids, the centers of mass of the binarized 'blobs' were computed.

A more sophisticated routine, such as fitting an ellipse to the boundary of the aluminum bars, would surely yield increased centroid finding accuracy.

\subsection{Reconfiguration of Fiducial Motifs}

In order to find the transformation from the holder coordinate system to the image space, ${ }_{H}^{I} T$, we find three corresponding points in both the holder and image coordinate systems. Upon examining our data, we find that the accuracy in determining the holder orientation is dependent upon the distance between these three points. That is, a set of points that are widely separated will produce a more accurate orientation than a set of closely placed points. In order to demonstrate this, we plot the angular error versus the shortest 'moment arm' of the three points (Figure 4). The moment arm is defined as the perpendicular distance from one point to the line connecting the other two points. We find that there is a significant dependence of angular error on the shortest moment arm ( $p$ value of 0.02 ).

With a simple reconfiguration of the localization module (i.e. reversing the base plate orientation) we can ensure that the smallest possible moment arm is $67 \mathrm{~mm}$. This is a significant increase over the present configuration, where the shortest moment arms vary from $15 \mathrm{~mm}$ to $47 \mathrm{~mm}$. With an increase in distance between corresponding points, we can expect an increase in angular accuracy.

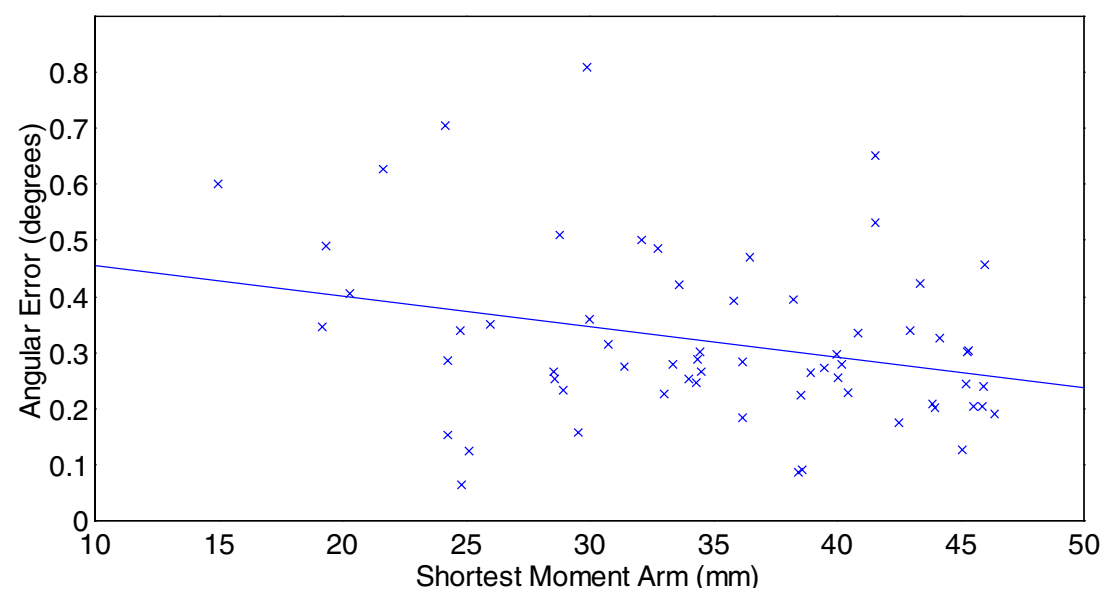

Figure 4: Angular error is a function of the shortest 'moment arm'. The $p$ value for the linear regression is 0.02 . With minor modification of our localization module, we can increase the shortest possible moment arm to $67 \mathrm{~mm}$. 


\subsection{Adaptation/Extension to MRI}

We note that with minor changes in materials, our localization system can be easily extended for applications using magnetic resonance imaging. With fast spin echo MRI, which allows for rapid image acquisition (e.g. 'MR fluoroscopy'), both the patient's anatomy and our end effector could be followed in real time, allowing for accurate and interactive effector guidance in a variety of percutaneous procedures.

\section{References}

1. Jain, R.K., The Next Forntier of Molecular Medicine: Delivery of Therapeutics. Nature, 1997(4): p. 655-657.

2. Klausner, R.D., The Nation's Investment in Cancer Research: A Budget Proposal for Fiscal Year 1999, . 1997, National Cancer Institute.

3. Galloway, R.L., Stereotactic Frame Systems and Intraoperative Localization Devices, in Interactive Image-guided Neurosurgery, R.J. Maciunas, Editor. 1993, American Association of Neurological Surgeons. p. 9-16.

4. Brown, R.A., A stereotactic head frame for use with CT body scanners. Invest. Radiol., 1979. 14: p. 300-304.

5. Brown, R.A., T.S. Roberts, and A.G. Osborne, Stereotaxic frame and computer software for CT-directed neurosurgical localization. Invest. Radiol., 1980. 15: p. 308-312.

6. Clarke, R.H. and e. al., On the intrinsic fibers of the cerebellum, its nuclei, and its efferent tracks. Brain, 1905. 28: p. 12-29.

7. Goerss, S., et al., A computed tomographic stereotactic adaptation system. Neurosurgery, 1982. 10: p. 375-379.

8. Leksell, L. and B. Jernberg, Stereotaxis and tomography: a technical note. Acta Neurochir., 1980. 52: p. 1-7.

9. Mundinger, F., CT-stereotactic biopsy of brain tumors, in Tumors of the Central Nervous System in Infancy and Childhood, D. Goth, P. Gutjahr, and C. Langmaid, Editors. 1982, Springer-Verlag: New York. p. 234-246.

10. Lavallee, S., et al., Image-Guided Operating Robot: A Clinical Application in Stereotactic Neurosurgery, in Computer-Integrated Surgery, R.H. Taylor, et al., Editors. 1996, MIT Press: Cambridge, Mass. p. 343-352.

11. Kwoh, Y.S., Hou. J., and E.A. Jonckheere, et. al., A robot with improved absolute positioning accuracy for CT guided stereotactic brain surgery. IEEE Trans Biomed Eng, 1988. 35(2): p. 153-161.

12. Glauser, D., et al. Neurosurgical Robot Minerva, First Results and Current Developments. in Proc. 2nd Int. Symp. on Medical Robotics and Computer Assisted Surgery. 1995. Baltimore, Maryland: MRCAS '95 Symposium, C/O Center for Orthop Res, Shadyside Hospital, Pittsburgh, Pa.

13. Masamune, K., et al. A newly developed stereotactic robot with detachable driver for neurosurgery. in Proc. 2nd Int. Symp. on Medical Robotics and Computer Assisted Surgery (MRCAS). 1995. Baltimore, Md.: MRCAS '95 Symposium, C/O Center for Orthop Res, Shadyside Hospital, Pittsburgh, Pa.

14. Bzostek, A., et al. An automated system for precise percutaneous access of the renal collecting system. in Proceedings the First Joint Conference of CVRMed and MRCAS. 1997. Grenoble, France.

15. Schreiner, S., et al. A system for percutaneous delivery of treatment with a fluoroscopically-guided robot. in Joint Conf. of Computer Vision, Virtual Reality, and Robotics in Medicine and Medical Robotics and Computer Surgery. 1997. Grenoble.

16. Stoianovici, D., et al. An efficient needle injection technique and radiological guidance method for percutaneous procedures. in First Joint Conference: CRVMed II \& MRCAS III, March. 1997. Grenoble, France.

17. Stoianovici, D., et al. A Modular Surgical Robotic System for Image-Guided Percutaneous Procedures. in Medical Image Computing and Computer-Assisted Interventions (MICCAI98). 1998. Cambridge, Mass: Springer. 